\title{
Trigonelline Extends the Lifespan of $C$. Elegans and Delays the Progression of Age-Related Diseases by Activating AMPK, DAF- 16, and HSF-1
}

\author{
Wen-Yu Zeng, ${ }^{1}$ Lin Tan, ${ }^{2}$ Cong Han, ${ }^{1}$ Zhuo-Ya Zheng, ${ }^{2}$ Gui-Sheng Wu, ${ }^{2,3}$ \\ Huai-Rong Luo $\mathbb{D}^{2,3}$ and Su-Lian Li ${ }^{1}{ }^{1}$ \\ ${ }^{1}$ Affiliated Traditional Chinese Medicine Hospital of Southwest Medical University, Luzhou, Sichuan 646000, China \\ ${ }^{2}$ Key Laboratory for Aging and Regenerative Medicine, Department of Pharmacology School of Pharmacy, \\ Southwest Medical University, 319 Zhongshan Road, Luzhou, Sichuan 646000, China \\ ${ }^{3}$ Central Nervous System Drug Key Laboratory of Sichuan Province, Luzhou, Sichuan 646000, China
}

Correspondence should be addressed to Huai-Rong Luo; lhr@swmu.edu.cn and Su-Lian Li; lyzyyhlb@163.com

Received 16 July 2021; Revised 19 August 2021; Accepted 27 August 2021; Published 26 September 2021

Academic Editor: Stefania D’Adamo

Copyright (C) 2021 Wen-Yu Zeng et al. This is an open access article distributed under the Creative Commons Attribution License, which permits unrestricted use, distribution, and reproduction in any medium, provided the original work is properly cited.

\begin{abstract}
Trigonelline is the main alkaloid with bioactivity presented in fenugreek, which was used in traditional medicine in Asian countries for centuries. It is reported that trigonelline has anti-inflammatory, anti-oxidant, and anti-pathogenic effects. We are wondering whether trigonelline have anti-aging effect. We found that $50 \mu \mathrm{M}$ of trigonelline had the best anti-aging activity and could prolong the lifespan of Caenorhabditis elegans (C. elegans) by about $17.9 \%$. Trigonelline can enhance the oxidative, heat, and pathogenic stress resistance of $C$. elegans. Trigonelline could also delay the development of neurodegenerative diseases, such as $\mathrm{AD}, \mathrm{PD}$, and $\mathrm{HD}$, in models of $C$. elegans. Trigonelline could not prolong the lifespan of long-lived worms with lossof-function mutations in genes regulating energy and nutrition, such as $c l k-1$, isp-1, eat-2, and $r s k s-1$. Trigonelline requires daf$16, h s f-1$, and aak-2 to extend the lifespan of C. elegans. Trigonelline can also up-regulate the expression of $d a f-16$ and $h s f-1$ targeted downstream genes, such as sod-3, gst-4, $h s p-16.1$, and $h s p-12.6$. Our results can be the basis for developing trigonelline-rich products with health benefits, as well as for further research on the pharmacological usage of trigonelline.
\end{abstract}

\section{Introduction}

The percentage of people aged 65 years and older in developed and developing countries has been steadily increasing. Aging is an intrinsic feature of life and is the greatest risk factor for major age-related disorders, such as diabetes, hypertension, cardiovascular disease, cancer, and neurodegenerative disease [1]. The prevalence of the older population has led to a global burden of age-related disorders. For many years, people have been looking for substances to prevent aging. Experimenting on mammals is time-consuming and expensive. Researchers use cultured human cells to test the anti-aging activity of more substances, but aging is a complex process that is difficult to measure at the cellular level. Caenorhabditis elegans (C. elegans) is the only multicellular model organism whose somatic developmental lineages have been clearly studied. C. elegans has a short life cycle and simple structure. C. elegans also has clear genetic background and is easy to manipulate. So, it has become an ideal model for aging research, as well as anti-aging drug screening.

Fenugreek (Trigonella foenum-graecum L.) is an annual forage legume and a traditional spice crop and has been used in traditional medicine in Asian countries for centuries [2]. Modern pharmacological studies have shown that fenugreek has antidiabetic, anticarcinogenic, hypocholesterolemic, antioxidant, and immunological activities [3]. Trigonelline (TRG) is one of the main alkaloids and components with pharmacological activities in dried fenugreek seeds (Figure 1(a)). Trigonelline has been reported to have neuroprotective $[4,5]$, anti-apoptotic $[6,7]$, anti-inflammatory $[8,9]$, anti-oxidant $[10]$, anti-diabetic $[11,12]$, antihyperglycemic effects [13], anti-degranulation [14], and anti- 


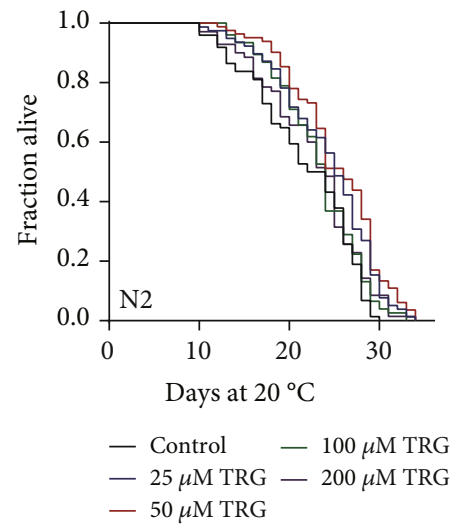

(a)

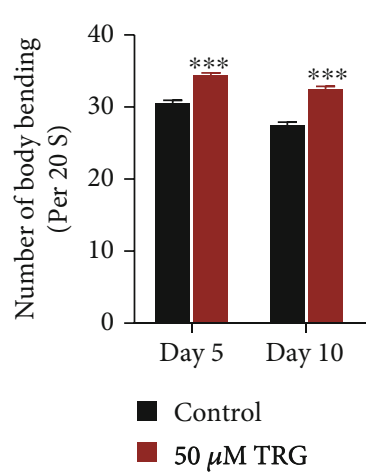

(d)

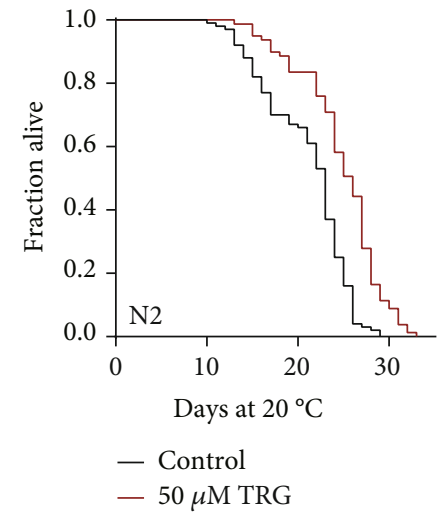

(c) (b)

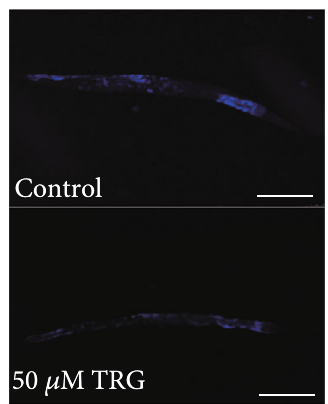

$50 \mu \mathrm{M}$ TRG

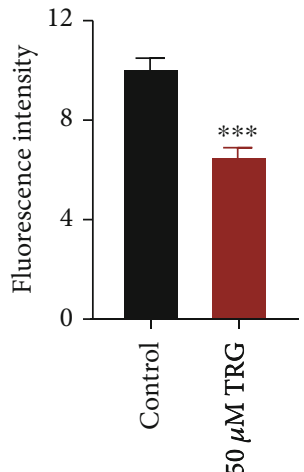

(e)

Figure 1: Trigonelline can prolong the lifespan and improve the health of C. elegans (a) Chemical structure of trigonelline; (b) Survival curves of wild-type $\mathrm{N} 2$ worms raised at $20^{\circ} \mathrm{C}$ on NGM plates containing either no trigonelline or $25,50,100$, and $200 \mu \mathrm{M}$ of trigonelline; (c) Survival curves of wild-type $\mathrm{N} 2$ worms treated from hatching and raised at $20^{\circ} \mathrm{C}$ on NGM plates containing either no trigonelline or $50 \mu \mathrm{M}$ of trigonelline $(p<0.001, \log$-rank test), Statistical details and repeats of these experiments were summarized in Table S1; (d) Body bending of $\mathrm{N} 2$ nematode treated with $50 \mu \mathrm{M}$ of trigonelline for 5 days and 10 days. Figure shows the mean value of three independent experiments, SEM is represented by error line. $* * *$ represents $p<0.001$, calculated by two-tailed t-test. Statistical details and repeats of these experiments were summarized in Table S3; (e) Analysis of lipofuscin in Treatment of N2 nematodes by 50 of $\mu \mathrm{M}$ trigonelline on day 10. Relative Fluorescence Intensity was calculated by Image J. Figure shows the mean value of three independent experiments, SEM is represented by error line. $* * *$ represents $p<0.001$, calculated by two-tailed t-test. Statistical details and repeats of these experiments were summarized in Table S2.

carcinogenic effect $[15,16]$. Among them, the anti-oxidative activity of trigonelline could explain many of its other activities, such as neuroprotective activity [3]. The antioxidant and neuroprotective effects of trigonelline lead us to speculate that trigonelline may play an important role in anti-aging and treating neurodegenerative diseases.

In this study, we investigated whether trigonelline could extend the lifespan and increase the stress tolerances of $C$. elegans. We also tested whether trigonelline could delay the onset of neurodegenerative disease in models of C. elegans, as well as determine its possible mechanisms.

\section{Materials and Methods}

2.1. Materials. The trigonelline was supplied by Shanghai Yuanye Bio-Technology Ltd (Shanghai,

China, purity $\geq 95 \%$ ). Other compounds used in this work were purchased from Sigma-Aldrich (Munich, Germany).
Trigonelline was dissolved in $\mathrm{ddH}_{2} \mathrm{O}$. NGM plates with compound were equilibrated overnight before use.

2.2. Worm Strains. All worm strains were provided by the Caenorhabditis Genetic Center (CGC, University of Minnesota, Minneapolis, MN). Except for special cases, worms were cultured in a constant temperature and humidity incubator with a temperature of $20^{\circ} \mathrm{C}$ and humidity of $60 \%$, on a medium with OP50 E. coli food. The nematode strains were as follows: N2 (wild-type), CF1903 glp-1(e2144) III., CF1038 daf-16 (mu86) I., EU1 skn-1(zu67)IV., PS3551 hsf-1(sy441)I., DA1116 eat-2(ad1116) II., MQ887 isp-1(qm150) IV., CB1370 daf-2(e1370) III., RB754 aak-2(ok524)X., RB759 akt1(ok525)V., VC204 akt-2(ok393)X., VC199 sir-2.1(ok434)IV., CB4876 clk-1(e2519)III., TK22 mev-1(kn1)III., BX165 nhr80 (tm1011)., AA89 daf-12 (rh274)., BZ555 egIs1(dat-1::gfp), CF1553 [(pAD76) sod-3::GFP + rol6 (su1006)], CL2166, dvIs19[pAF15(gst-4::GFP::NLS)], SJ4100 (zcIs13[hsp6::GFP]), SJ4005 zcIs4V (hsp-4::gfp), SJ4058 zcIs9 [hsp- 
60::GFP + lin-15(+)], CL4176 [(pAF29)myo-3p::A $\beta 1-42+$ (pRF4) rol-6 (su1006)], CL2006 (dvIs2 [pCL12(unc54/human A $\beta 1-42$ minigene) + pRF4]), NL5901 [unc54p::alphasynuclein::YFP + unc-119(+)], AM140 (rmIs132[unc54p::Q35::YFP]).

2.3. Lifespan Assay. All strains were cultured for 2 - 3 generations without starvation on new NGM plates.

The worms were transferred to NGM plates (containing inactivated OP50 $\left(60^{\circ} \mathrm{C}\right.$ for $\left.\left.35 \mathrm{~min}\right)\right)$ with or without trigonelline in late L4 or early adult stage. The plates were supplemented with $20 \mu \mathrm{M}$ of 5-fluoro-2'-deoxyuridine (FUdR) to inhibit oviposition and hatching. The day when Caenorhabditis elegans was transferred to the plates was recorded as the 0th day of the experiment [17]. To ensure that trigonelline retained its potency throughout the entire experiment, worms were transferred to fresh plates with or without trigonelline every other day. In the statistics of lifespan test, if nematodes did not move or did not respond to external mechanical stimulation (such as touching them lightly with insect pickers), they would be recorded as dead. The statistical data of experiments were processed by SPSS 20.0 software, and the results were expressed by Kaplan-Meier survival curve. The $p$ value was obtained by log-rank test analysis, while $p<0.05$ indicates statistical significance. Each group of lifespan experiments included at least three independent repeated experiments, and the number of worms in each group was more than 60 .

2.4. Stress Resistance Assay. For the high temperature resistance test, the synchronized larvae were spread on NGM plates, cultured in an incubator at $20^{\circ} \mathrm{C}$, and then transferred to NGM plates with or without trigonelline at the late L4 stage or early adult stage. The worms were transferred to $35^{\circ} \mathrm{C}$ on the 10 th day of adult, and the death of the worm was observed and counted every 2 hours.

For anti-oxidative stress test, pretreatment was the same as high temperature test, the adult worms on the 10th day are transferred to NGM plates containing $20 \mathrm{mM}$ of paraquat, and the death of worms is observed and counted every other day.

For the pathogenic stress test, the pre-treatment was the same as high temperature resistance test. On the 10th day of the adulthood, the worms were transferred to the NGM plates covered with bacteria Pseudomonas aeruginosa [17]. The death of worms was observed and recorded every 12 hours.

All statistics and analysis methods of stress assays were the same as the lifespan experiment. The sample size of each experiment was at least 60 worms, and each stress assay contains at least three independent repeated experiments.

2.5. Body Bending Behavior Test. The synchronized L1 larva nematodes were spread on NGM plates, cultured in incubator at $20^{\circ} \mathrm{C}$. the worms developed to $\mathrm{L} 4$ were transferred to experimental plate with or without trigonelline. On the 5th and 10th days of adulthood, the worms were picked up to water droplets and stabilized for 1 minute. Then the bending frequency of the body within 20 seconds were recorded under the microscope, and the back and forth movement of the body was calculated as one bend.

2.6. Determination of Reactive Oxygen Species (ROS). The L1 larvae were spread on NGM plate and cultured in an incubator at $20^{\circ} \mathrm{C}$ until late $\mathrm{L} 4$ or early adulthood. Then, worms were transferred to experimental plates (control group, Trigonelline, NAC, paraquat); After $96 \mathrm{~h}$, the worms were collected and stained with H2DCF-DA probe according to the procedure of ROS detection kit [18]. Then, the nematodes were photographed with fluorescence microscope. The ROS detection experiment includes at least three independent repeated experiments. The sample size of each experiment was at least 30 worms. Image J was used to count the gray value of each nematode. The $p$ value was calculated by two-tailed $t$-test.

2.7. Determination of Lipofuscin. The preliminary treatment was the same as the lifespan test, the nematodes were collected on the tenth day of the adult and photographed by fluorescence microscope at excitation wavelength of 360$370 \mathrm{~nm}$ and emission wavelength of $420-460 \mathrm{~nm}$; The level of lipofuscin in each nematode was quantified by calculating the average pixel intensity of each nematode. Sample size is at least 30 worms.

2.8. Fluorescence Quantification. The preliminary treatment of worms for fluorescence quantification was the same as the lifespan test, then the worms of strains Cl2166 (dvis19 [pgst4p::GFP::nls]) and CF1553 [(pad76) SOD-3::GFP + rol6 (su1006)] were placed at plates containing $20 \mathrm{mM}$ of paraquat for $30 \mathrm{~min}$ on the 6 th day of adulthood, anesthetized with $2 \mathrm{mM}$ of tetraimidazole hydrochloride. The expression of GFP in these strains was observed by fluorescence microscope. For Transgenic strains SJ4100 (zcIs13[hsp-6::GFP]), SJ4005 zcIs4V (hsp-4::gfp), and SJ4058zcis9 [HSP-60::GFP + Lin-15 $(+)]$, worms were placed at $35^{\circ} \mathrm{C}$ for $2 \mathrm{~h}$ on the 6 th day of adults, and was collected with a $1.5 \mathrm{~mL}$ centrifuge tube, washed with $\mathrm{ddH}_{2} \mathrm{O}$ for three times to remove OP50, anesthetized with $2 \mathrm{mmol}$ tetraimidazole hydrochloride, and observed the expression of green fluorescent protein with fluorescence microscope.

2.9. Age-Related Neurodegenerative Diseases Related Experiments. For worms of strain CL4176(dvis27 (myo3/abeta1-42/letutr) + prf4 (rol-6 (su1006)), the synchronized larva were spread on the NGM plates with or without trigonelline, incubated at $15^{\circ} \mathrm{C}$ for 36 hours, then transferred to $23^{\circ} \mathrm{C}$ for 24 hours, and then counted and photographed every 12 hours.

For worms of strain CL2006 (dvIs2 [pCL12(unc54/human A $\beta 1-42$ minigene) + pRF4]), the synchronized larvae were spread on NGM plates until they grow into late L4 or early adult stage. Then, the worms were transferred to NGM plate with or without trigonelline, and counted and photographed every day. Nematodes that remain immobile or move only by the head under external mechanical stimulation (such as touching with an insect picker) were defined as paralyzed. The number of worms per group should be above 60 . 
For the worms of strain AM40 and NL5901, the late L4 larva or early adulthood worms were transferred to experimental plates with or without trigonelline. AM140 nematodes were collected on the 2 nd and 4th day of adulthood, and NL5901 nematodes were collected on the 10th day of adulthood. Then, worms were anesthetized with $2 \mathrm{mM}$ of tetraimidazole hydrochloride. The aggregation of $\alpha$-synuclein was captured by Leica fluorescence microscope and analyzed by Image J. The experiment was independently repeated at least three times. The number of worms in each group of experiment is at least 30 .

For the worms of transgenic strain BZ555, the L3 larva were transferred to a centrifuge tube containing a mixture of $50 \mathrm{mM}$ of 6 -OHDA and $10 \mathrm{mM}$ of ascorbic acid and incubated at $20^{\circ} \mathrm{C}$ for one hour, and gently shaken every 10 minutes to mix. After one hour, the worms were washed three times with M9 and then placed on a culture plate with and without trigonelline for 72 hours. Finally, we washed the worms with M9, took pictures, and calculated statistics. The experiments were repeated independently at least twice. The number of worms in each group of experiment was at least 30 .

2.10. Quantitative RT-PCR Assay. About 3,000 synchronized young adult worms were transferred to NGM plates with or without $50 \mu \mathrm{M}$ of trigonelline and cultured at $20^{\circ} \mathrm{C}$ for 24 hours. Total RNA was extracted by RNAiso Plus (Takara), converted to cDNA using High Capacity cDNA Reverse Transcription Kit (Applied Biosystems). The quantitative RT-PCR was performed using Power SYBR Green PCR Master Mix (Applied Biosystems) on Quantstudio 6 Flex system. The relative expression levels of genes were calculated by the $2^{-\Delta \Delta \mathrm{Ct}}$ and normalized to the expression of gene cdc-42. The $p$ values were calculated using t-test.

\section{Results}

3.1. Trigonelline Can Prolong the Lifespan of C. Elegans. To investigate whether trigonelline have anti-aging activity, we treated wild-type N2 worms with $0,25,50,100$, and $200 \mu \mathrm{M}$ of trigonelline, respectively. Our results showed that trigonelline can extend the lifespan of C. elegans under various concentrations, among which, $50 \mu \mathrm{M}$ of trigonelline has the best effect on lifespan extension (Figures 1(b)-1(c)). Lipofuscin is a yellow-brown pigment that accumulates with age [19]. Our results showed that trigonelline could decrease the deposition of lipofuscin (Figure 1(e)). The movement of worms declines as age increases. We found that trigonelline treatment could enhance the movement of worms both at day 5 and day 10 of adulthood (Figure 1(d)).

3.2. Trigonelline Can Enhance the Stress Resistance of $C$. Elegans. Genetic and dietary interference that extend lifespan usually could also enhance the stress resistance of $C$. elegans [20]. So, we investigated if trigonelline could increase the resistance of $C$. elegans to oxidative, heat, and immune stresses. we detected the changes of ROS content in N2 treated with trigonelline. Trigonelline significantly reduced ROS level in nematodes, and the effect was similar to the antioxidant NAC (Figure 2(d)). Moreover, trigonelline increased the survival rate of $\mathrm{N} 2$ worms under oxidative stress induced by $20 \mathrm{mM}$ of paraquat (Figure 2(a)). Trigonelline treatment significantly increased the mRNA and protein expression levels of genes encoding superoxide dismutase 3 (SOD-3) and glutathione S-transferase 4 (GST4) (Figures 2(b)-2(c)). These results suggest that trigonelline had strong antioxidant effects.

Trigonelline can enhance the survival of $C$. elegans under high temperature of $35^{\circ} \mathrm{C}$ (Figure $3(\mathrm{a})$ ). Our results showed that trigonelline could increase the mRNA levels of heat shock protein encoding genes, such as $h s p-4, h s p-60$, and $h s p-6$ (Figure $4(\mathrm{~g})$ ). trigonelline treatment could also increase the fluorescence intensity of transgenic nematode strains expressing HSP-4::GFP or HSP-6::GFP (Figures 3(b)-3(d)).

Our results also showed that the survival of N2 nematodes in pathogenic bacteria PA14 was prolonged after being treated with trigonelline for 10 days. Trigonelline treatment increased the mRNA expression levels of immune-related genes, such as T24B8.5, F08G5.6, F35E12.5, F55G11.4 and irg-1 (Figures 3(e)-3(f)).

3.3. Trigonelline Can Delay the Progression of Age-Related Diseases in C. Elegans Models of AD, PD, and HD. Neurodegenerative diseases are characterized by chronic and progressive decline in neuronal function, which in turn leads to memory deficits, cognitive decline and motor coordination disorder [21]. These diseases include Alzheimer's disease (AD), Parkinson's disease (PD), Huntington's disease (HD), amyotrophy lateral sclerosis (ALS), and spinal muscular atrophy Disease (SMA).

The worm CL4176 expresses the human polypeptide $\mathrm{A}_{\beta 1-42}$ in the body wall muscle cells at the ambient temperature, resulting in paralysis phenotype of $\mathrm{AD}$-like symptoms in worms. Our results show that trigonelline treatment could delay the onset of paralysis in CL4176 worms (Figure 5(c)). The worm CL2006 also expresses human $\mathrm{A}_{\beta 1-42}$ driven by the promotor of gene unc-54 that encoding actin filament. Adult of CL2006 onset paralysis and egg-laying deficiency when raised at 20C. Our results showed that the survival of the nematode CL2006 under progressive paralysis were prolonged after trigonelline treatment (Figures 5(d)-5(e)).

Parkinson's disease (PD) model of nematode NL5901 expresses a human $\alpha$-syn protein fused with yellow fluorescent protein (YFP) in muscle cells of body wall. Trigonelline significantly reduced the $\alpha$-syn aggregation (Figure 5(a)). Worms BZ555 expresses green fluorescent protein (GFP) in the somatic cells and axons of dopamine neurons. Treatment of Caenorhabditis elegans with 6-hydroxydopamine (6-OH DA) can selectively induces dopaminergic neuron degeneration. We found that trigonelline can recover neuron injury induced by 6-OH DA, and its activity is like that of the positive drug levodopa (Figure 5(b)).

Huntington's disease (HD) is a fatal autosomal dominant neurodegenerative disease. Its neuropathological features are progressive lesions in basal ganglia, and its clinical manifestations are abnormal movement, cognition, and behavior. AM140 is a transgenic strain expressing 


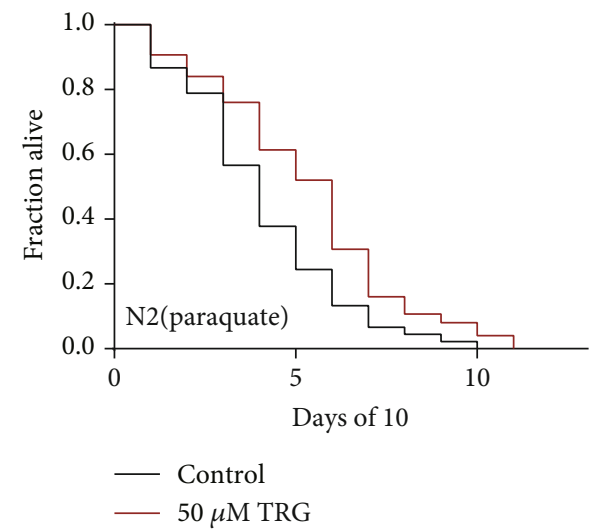

(a)

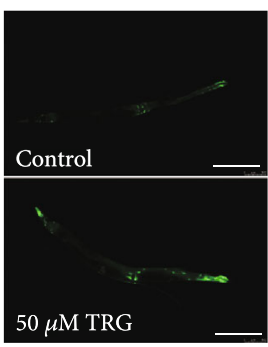

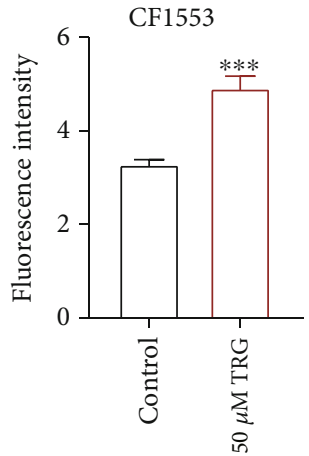

(b)
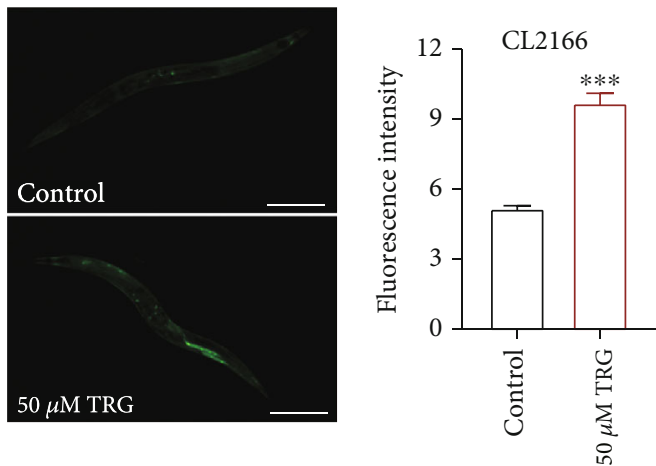

(c)

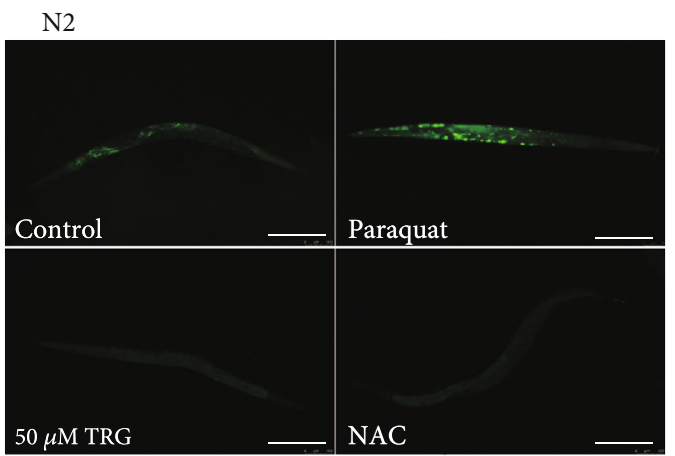

(d)

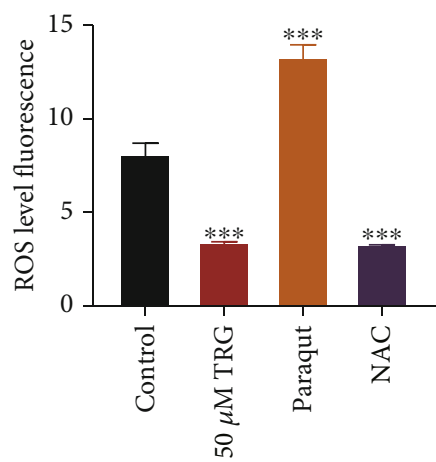

Figure 2: Trigonelline can enhance the oxidative stress resistance of $C$. elegans. The survival percentage of wild-type worms cultured with $20 \mathrm{mM}$ of paraquat under treatment with $50 \mu \mathrm{M}$ of trigonelline $(p<0.001, \log$-rank test). Statistical details and repeats of these experiments were summarized in Table S6; (B-C) The quantification of fluorescence intensity of SOD-3::GFP in CF1553 and GST-4::GFP in CL2166. Trigonelline significantly increased the expression of SOD-3 and GST-4, Fluorescence intensity was calculated by Image J. The bar chart shows the mean value of three independently repeated experiments, and the error line represents SEM. $* * *$ represents $p<0.001$, calculated by two-tailed t-test. Statistical details and repeats of these experiments were summarized in Table S5; (D) ROS content detection of wild type $\mathrm{N} 2$ treated with $50 \mu \mathrm{M}$ of trigonelline, while $10 \mathrm{mM}$ of paraquat and $2 \mathrm{mM}$ of NAC were used as negative control and positive control, respectively. Statistical details and repeats of these experiments were summarized in Table S7.

yellow fluorescent protein (YFP)-labeled polyglutamine (polyQ). We measured the effect of trigonelline on fluorescence intensity and punctate aggregation in AM140. Trigonelline significantly reduced the accumulation of agerelated polyQ in body wall muscles on day 2 and day 4 of adults (Figure 5(f)). Above results shows that trigonelline can improve the performance of worms in neurodegenerative diseases models.
3.4. Trigonelline Requires the Transcription Factor DAF16/FOXO to Extend the Lifespan of C. Elegans. The transcription factor DAF-16/FOXO plays a central role in the regulation of the stress resistance and longevity [22]. So, we examined if trigonelline could extend the lifespan of worms with loss-of-function mutation in daf-16. We found that trigonelline could not extend the lifespan of the loss-of-function mutant daf-16 (mu86) I (Figure 4(b)). 


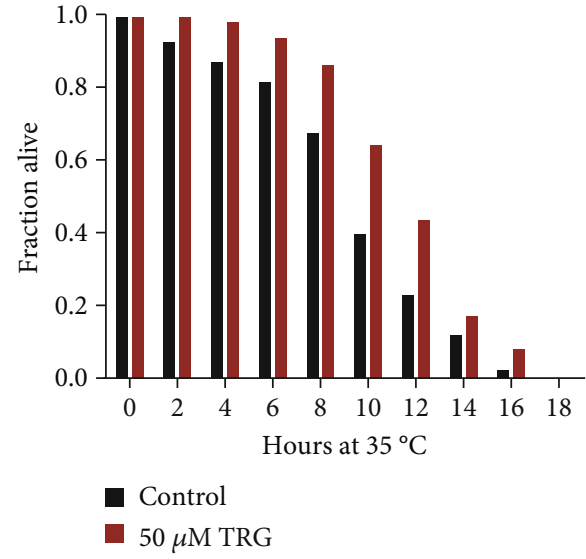

(a)

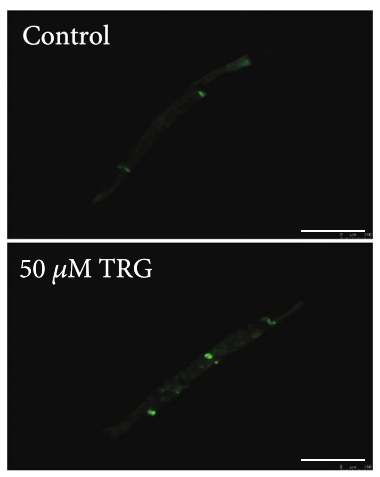

(c)

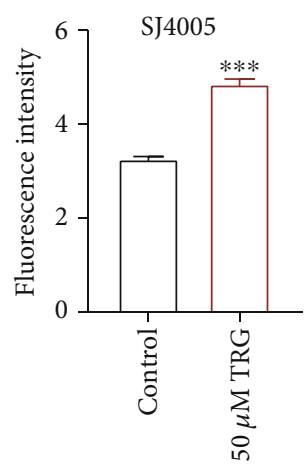

(c)

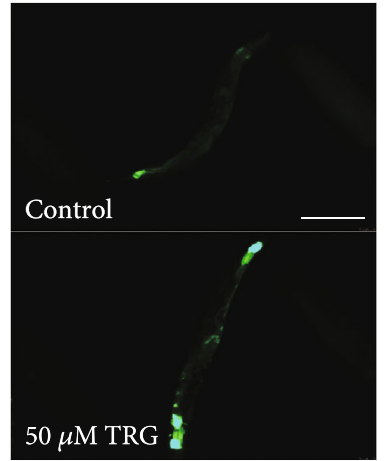

$50 \mu \mathrm{M}$ TRG

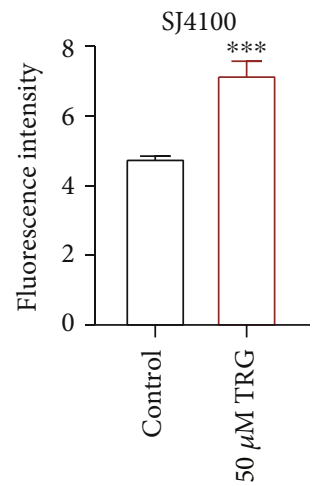

(b)

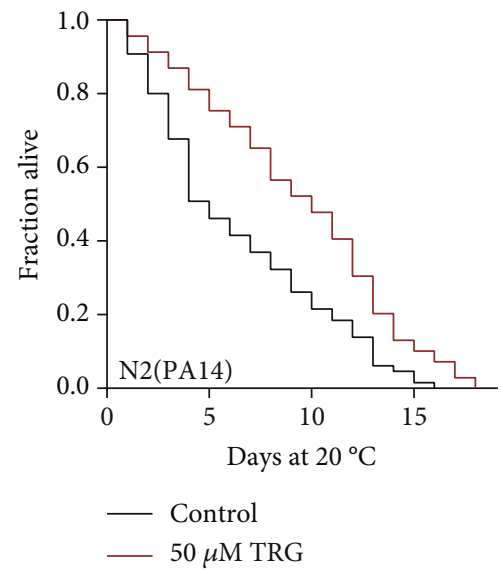

(e)
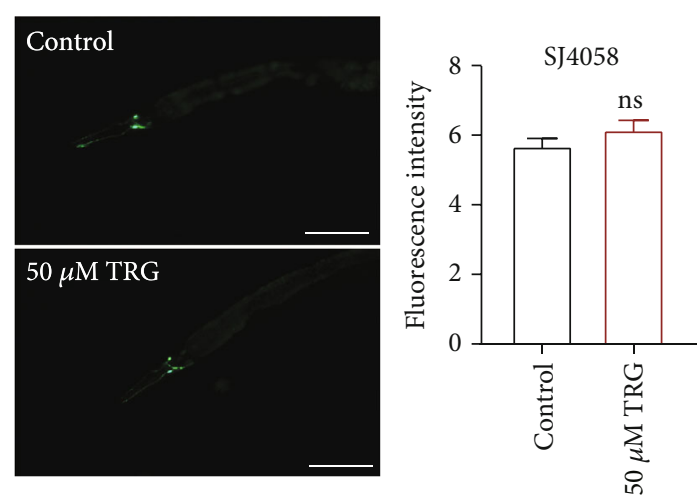

(d)

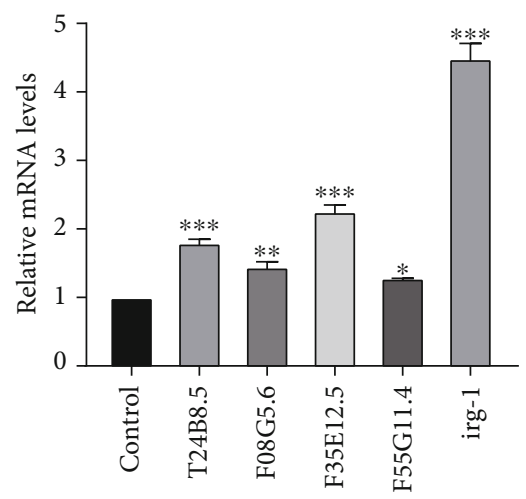

(f)

Figure 3: Trigonelline can enhance the ability of nematode to resist heat and pathogenic stresses (a) The survival percentage of wild-type worms treated with or without $50 \mu \mathrm{M}$ of trigonelline at $35^{\circ} \mathrm{C}(p<0.001)$, Statistic details and repeats of these experiments were summarized in Table S6; (B-D) The quantification of fluorescence intensity of HSP-6::GFP in SJ4100, HSP-4::GFP in SJ4005,HSP-60::GFP, and in SJ4058. Trigonelline significantly increased the expression of HSP-4 and HSP-6, Fluorescence intensity was calculated by Image J. The bar chart shows the mean value of three independently repeated experiments, and the error line represents SEM. $* * *$ represents $p<0.001$, calculated by two-tailed t-test. Statistical details and repeats of these experiments were summarized in Table S5; (E) The survival percentage of wild-type worms cultured with Pseudomonas aeruginosa under treatment with $50 \mu \mathrm{M}$ of trigonelline ( $p<0.001$, log-rank test). Statistical details and repeats of these experiments were summarized in Table S6; (F) Relative Expression of Immune Genes in L4 Wild-type Worm (N2) Treated with $50 \mu \mathrm{M}$ of trigonelline for $24 \mathrm{~h}$. Statistical details and repeats of these experiments were summarized in Table S9. 


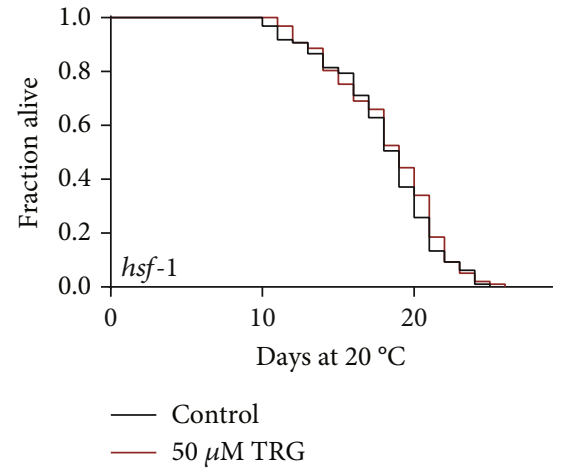

(a)

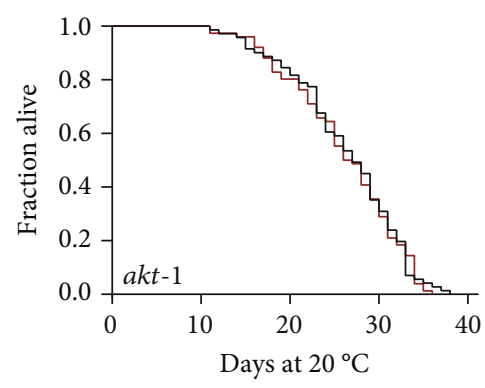

- Control
$-50 \mu \mathrm{M}$ TRG

(d)

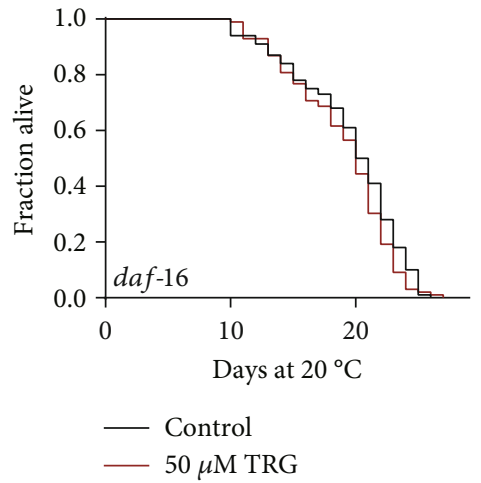

(b)
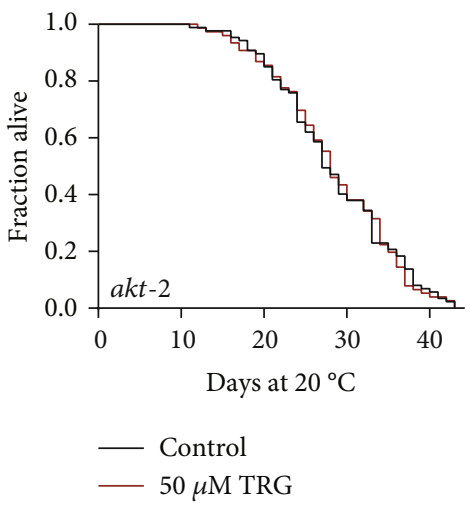

(e)

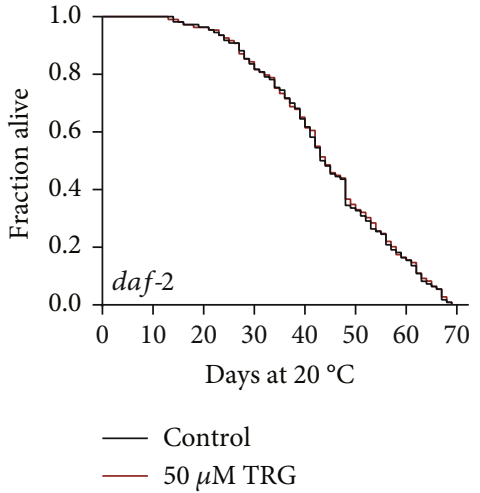

(c)

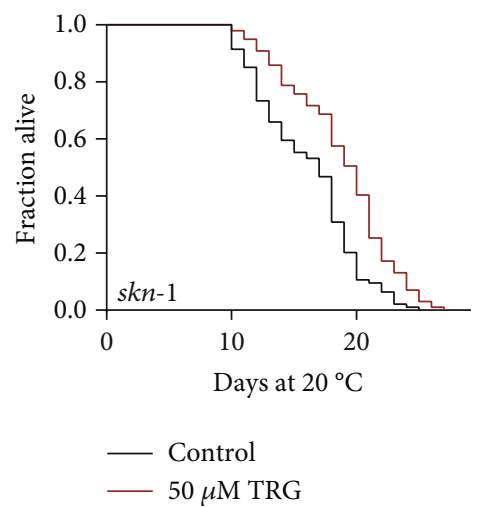

(f)

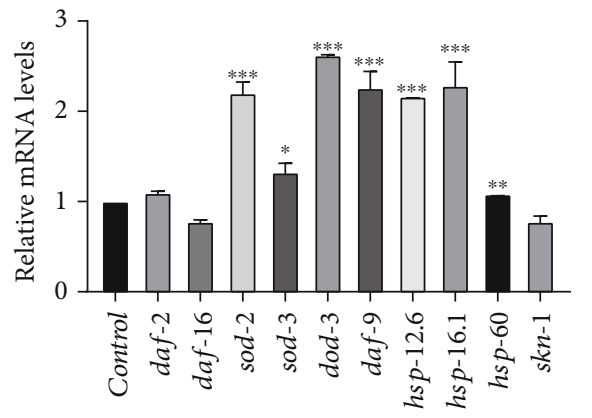

(g)

Figure 4: HSF-1 is required for trigonelline to extend nematode lifespan. (A-F) Survival curves of $h s f-1(s y 441) I$., daf-16(mu86)I., daf2(e1370)III., akt-1(ok525)V., akt-2(ok393)X., and skn-1(zu67), raised at $20^{\circ} \mathrm{C}$ on NGM plates containing either no trigonelline or $50 \mu \mathrm{M}$ of trigonelline in lifespan assays $(p>0.05)$. Lifespan was analyzed using Kaplan-Meier analysis and $p$ values were calculated using logrank test. Statistic details and repeats of these experiments were summarized in Table S4; (G) Relative expression of downstream daf-2 genes in L4 wild-type worms (N2) treated with $50 \mu \mathrm{M}$ of trigonelline for $24 \mathrm{~h}$. Statistical details and repeats of these experiments were summarized in Table S9.

The kinases AKT-1 and AKT-2 act at upstream of DAF-16 in the insulin signaling pathway. We found that trigonelline also could not extend the lifespan of worms with loss-of-function mutations in akt-1 and akt-2. (Figures $4(\mathrm{c})-4(\mathrm{e}))$.

The transcription factor SKN-1 is the homologue of mammalian NF-E2-related factor 2 (Nrf2), plays a central role in the induction of cytoprotective genes in response to oxidative stress [23]. We found that trigonelline could extend the lifespan of worms with a loss-of-function mutation in $s k n-1$ (Figure 4(f)).
The conserved heat stress transcription factor HSF-1 regulates the expression of cellular chaperone genes to maintain the proteostasis from external environmental stresses and internal age-related damages [24]. We investigated if trigonelline need HSF to extend the lifespan of worms. Our results showed that trigonelline could not extend the lifespan of worms with loss-of-function mutant in $h s f-1$ (Figure 4(a)).

3.5. Trigonelline Could Not Further Extend the Lifespan of Worms with Mutations in the Genes Regulating Energy Production and Nutrition Uptake. The decline in 


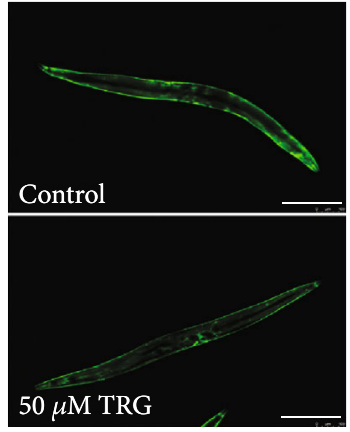

(a)

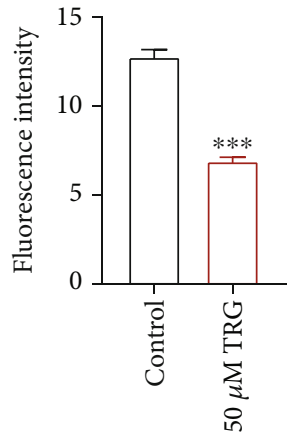

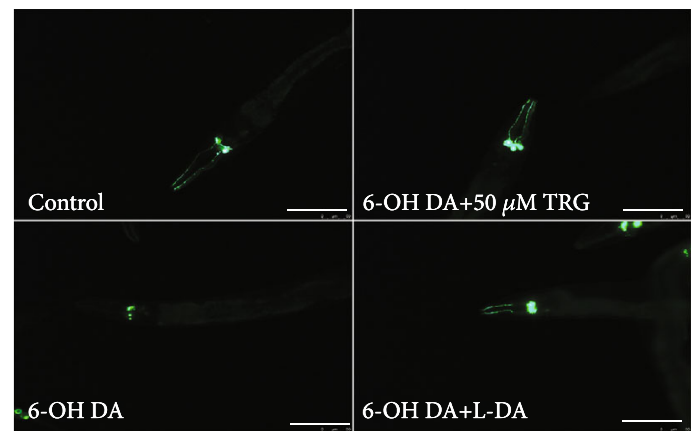

(b)

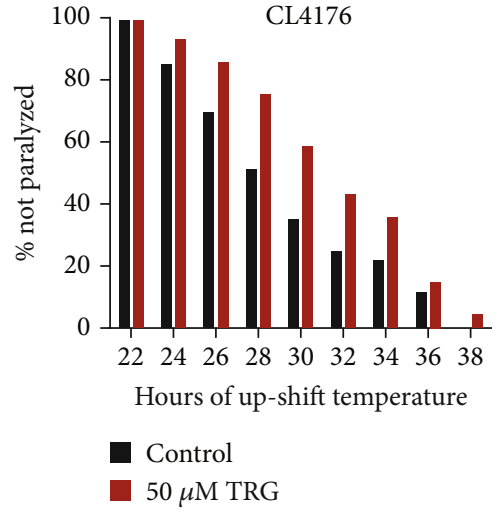

(c)

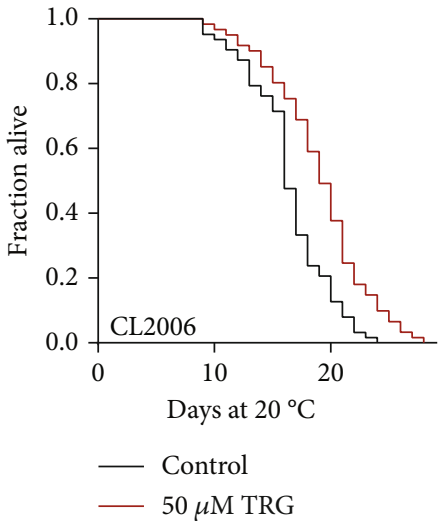

(d)
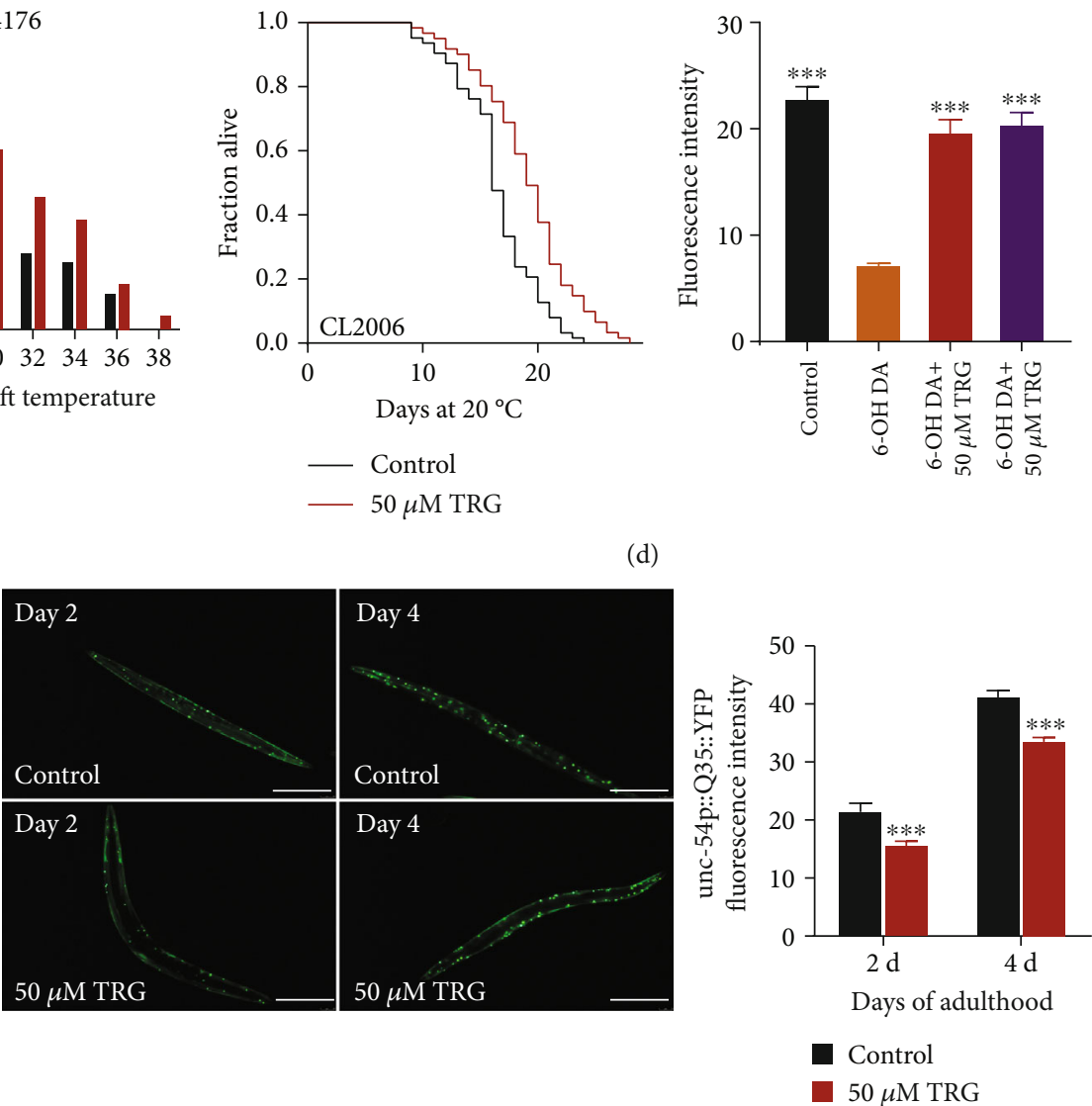

(f)

FIGURE 5: Trigonelline can delay the progression of age-related diseases in C. elegans models of AD, PD, and HD Effect of trigonelline on the accumulation of A-synuclein in PD model nematodes; (C-D) Effect of trigonelline on the paralysis rate of AD model in nematode strains CL4176 and CL2006; (E) Effect of trigonelline on the accumulation of Poly-Q in PD model nematodes on day 2 and day 4, Statistical details and repeats of these experiments were summarized in Table S8.

mitochondrial energy production and the damage caused by mitochondrial reactive oxygen species (ROS) are the main causes of aging [25]. The genes involved in mitochondrial electron transport are also the regulators of oxidative stress responses [26]. The gene $c l k-1$ encodes a mitochondrial enzyme or regulatory molecule in the ubiquinone biosynthetic pathway. The gene mev-1 encodes a homolog of the $\mathrm{C}$ subunit of the human succinate dehydrogenase complex, while isp- 1 encodes the ubiquitin-cytochrome $\mathrm{c}$ reductase, the homolog of Rieske iron-sulfur polypeptide 1 . We investigated if interruption of these genes would affect the effect of trigonelline on lifespan extension. Our results showed that trigonelline could not extend the lifespan of worms carrying the loss-of-function mutations of the three genes (Figures 6(a)-6(c)).

Dietary restriction can affect a variety of metabolic pathways and stress resistance pathways, including reducing cell damage induced by oxidative stress, improving mitochondrial function, thereby reducing age-related mitochondria function decline [27]. DA1116 eat-2(ad1116) II. has slowed pumping rate, mimics dietary restriction phenotypes and presents extended lifespan compared with wild type N2. 


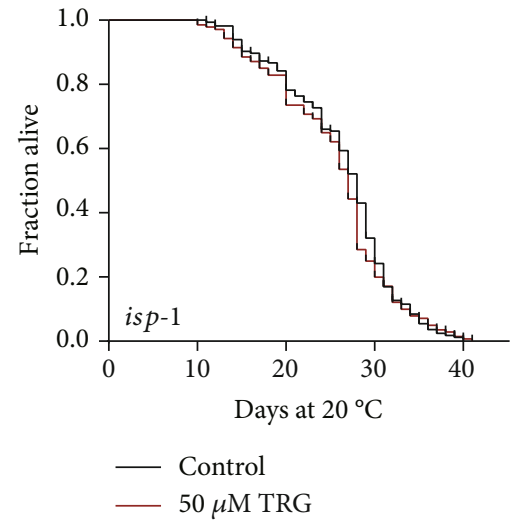

(a)

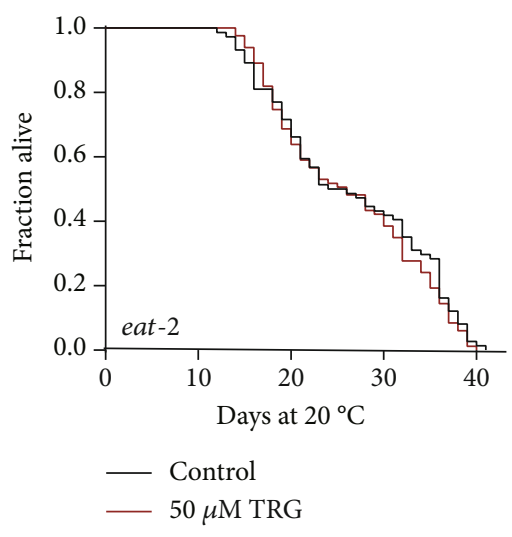

(d)

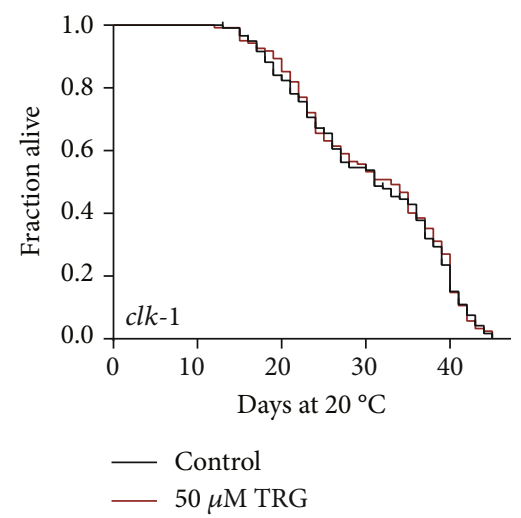

(b)

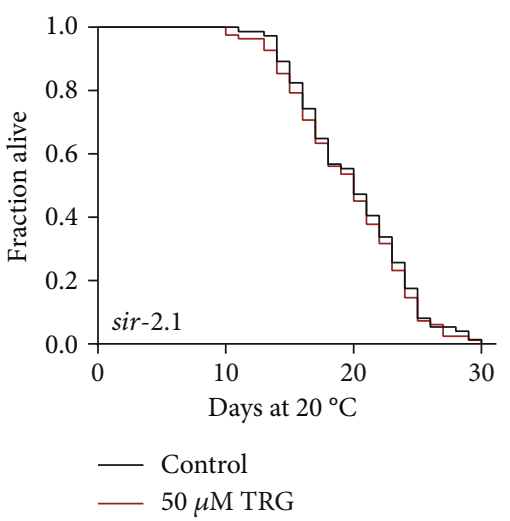

(e)

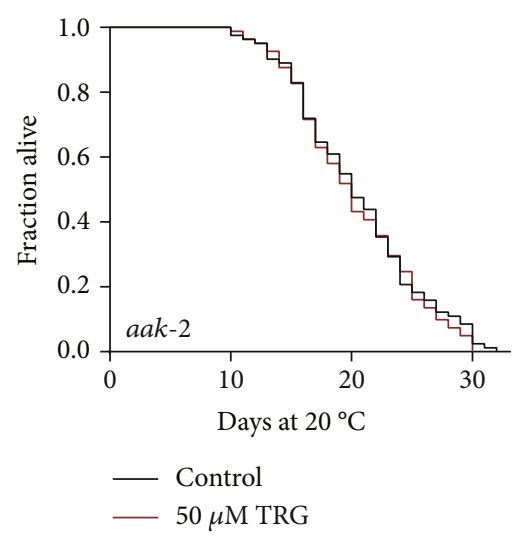

(g)

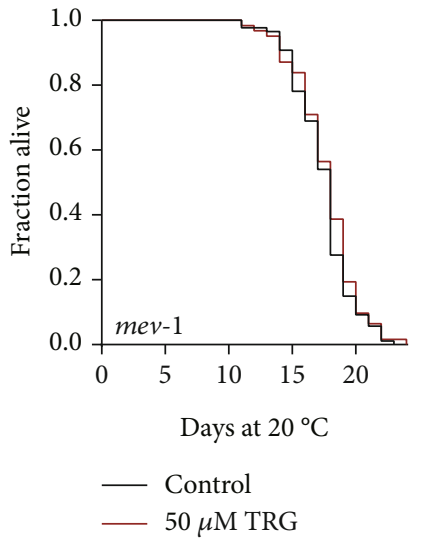

(c)

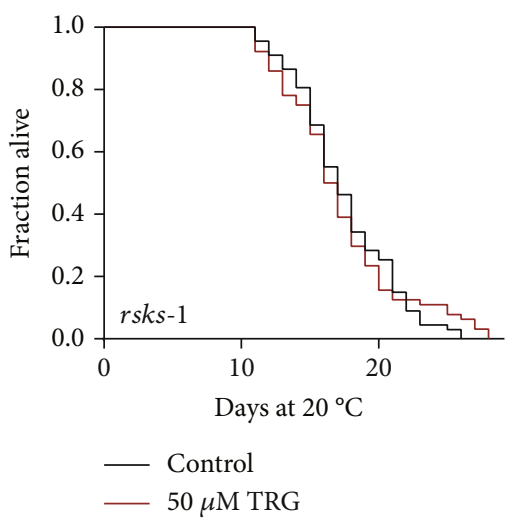

(f)

FIGURE 6: Trigonelline prolongs lifespan through mitochondrial signaling pathways (A-G) Survival curves of isp-1 (qm150), clk-1(e2519)III., and mev-1(kn1)III. raised at $20^{\circ} \mathrm{C}$ on NGM plates containing either no trigonelline or $50 \mu \mathrm{M}$ trigonelline in lifespan assays $(p>0.05)$. Lifespan was analyzed using Kaplan-Meier analysis and $p$ values were calculated using log-rank test. Statistic details and repeats of these experiments were summarized in Table S4.

We found that trigonelline could not extend the lifespan of DA1116 eat-2(ad1116) II.. Dietary restriction regulates the activity of NAD-dependent protein deacetylase SIR-2.1, 5'AMP-activated protein kinase (AMPK) catalytic subunit 2, and Ribosomal protein S6 kinase beta (encoded by gene rsks-1). We found that trigonelline treatment could not extend the lifespan of DA1116 eat-2(ad1116) II and worms with loss-of-function mutation in sir-2, which encodes NAD-dependent protein deacetylase (Figures 6(d)-6(e)).
Gene aak-2 encodes 5'-AMP-activated protein kinase catalytic subunit 2, which plays a role in the regulation of lifespan, dauer larval development and protein secretion. Ribosomal protein S6 kinase beta is a key factor of the target-of-rapamycin (TOR) pathway, regulates nematode lifespan and iron homeostasis. Our experiments show that trigonelline could not extend the lifespan of worms with loss-of-function mutations in genes aak-2 and rsks-1 (Figures 6(f)-6(g)). 


\section{Discussion}

Trigonelline, a plant alkaloid, is the main active ingredient of coffee and fenugreek $[2,28]$. We found that trigonelline at different concentrations can prolong the lifespan of C. elegans. Trigonelline treatment could enhance the locomotor ability of $C$. elegans, reduce the accumulation of lipofuscin in the body. Trigonelline can increase the heat, oxidative, and pathogenic stress resistance of worms. Trigonelline could also delay the progression of age-related diseases in C. elegans models of $\mathrm{AD}, \mathrm{PD}$, and $\mathrm{HD}$.

The transcription factor DAF-16/FOXO plays a central role in the regulation of the stress resistance and longevity [22]. The kinases AKT-1 and AKT-2 act at upstream of DAF-16 in the insulin signaling pathway [29]. The antiaging activity of trigonelline requires the transcription factor DAF-16/FOXO. Trigonelline also could not further extend the lifespan of worms with the loss-of-function mutations in $a k t-1$ and $a k t-2$. Since the mutants of akt-1 and akt-2 are long-lived, it was uncertain if trigonelline act on the upstream of AKT or the effect of Trigonelline on lifespan extension was not strong enough to show significant difference from the mutants of $a k t-1$ and akt-2. The transcription factor SKN-1plays a central role in the induction of cytoprotective genes in response to oxidative stress [23]. The conserved heat stress transcription factor HSF-1 regulates the expression of cellular chaperone genes to maintain the proteostasis [24]. Both SKN-1 and HSF-1 are the downstream target of DAF-16. Our results showed that trigonelline require HSF-1, but not SKN-1 to extend the lifespan of $C$. elegans, indicating trigonelline might act through stabilizing the proteostasis of C. elegans.

We found that trigonelline could not extend the lifespan of the worms with loss-of-function mutations in the genes clk-1, mev-1, isp-1, eat-2, sir-2.1, aak-2, and rsks-1. The genes $c l k-1, m e v-1$, and isp-1 encode mitochondrial proteins regulating energy production, while eat-2 regulates food uptake. AMPK senses the energy level and passes the information to TOR, which regulates the activity of ribosomal protein S6 kinase beta [27]. TOR is the central energy and nutrition sensor and plays a critical role in growth, metabolism, autophagy, and proteostasis [30]. Above results showed that trigonelline requires $a a k-2$ and extends the lifespan of C. elegans through the energy pathway.

A common etiology of neurodegenerative diseases, such as $\mathrm{AD}, \mathrm{PD}$, and $\mathrm{HD}$, is the abnormal deposition of proteins [31]. Our results showed that trigonelline could delay the onset of neurodegenerative diseases in various models of $C$. elegans. Trigonelline could delay the paralysis rate of Alzheimer's disease, reduce the accumulation of $\alpha$-syn in the worm muscle of Parkinson's model, repair the 6-OH DA-induced neuronal damage, and reduce the accumulation of Poly Q in AM140 worm of Huntington model. These results showed that trigonelline could improve the proteostasis of $C$. elegans. This is consistent with that trigonelline requires HSF-1 and AMPK for anti-aging effects, because both HSF-1 and AMPK could regulate the protein homeostasis of $C$. elegans.

In summary, trigonelline treatment can prolong the healthy lifespan, increase the stress tolerance of C. elegans, and delay the development of neurodegenerative diseases in models of nematodes. Finally, trigonelline appear to require transcription factor DAF-16/FOXO, HSF-1 and AMPK to maintain the protein homeostasis and extend the lifespan of C. elegans. Our results can be the basis for developing trigonelline-rich products with health benefits, as well as for further research on the pharmacological usage of trigonelline.

\section{Data Availability}

All the figures and tables used to support the findings of this study are included within the article and supplementary materials.

\section{Conflicts of Interest}

The authors declare that there is no conflict of interest.

\section{Authors' Contributions}

Wen-Yu Zeng and Lin Tan have contributed equally to this work.

\section{Acknowledgments}

Financial supports were received from the Natural Science Foundation of China (81771516), Central Nervous System Drug Key Laboratory of Sichuan Province (200014-01SZ and 200016-01SZ), Cooperation Project of Luzhou City Hospital of traditional Chinese medicine and Southwest Medical University (2019-LH005), and the Science and Technology Cooperation Project of Luxian and Southwest Medical University (2019LXXNYKD-04).

\section{Supplementary Materials}

Table S1. Lifespan of wild-type nematode (N2) treated with TRG in different concentrations. Table S2. Effect of TRG on lipofuscin in wild-type nematode (N2). Table S3. Effect of TRG on body movement in wild-type nematode (N2). Table S4. Effect of TRG on lifespan of mutant nematode. Table S5. Effect of TRG on DAF-16 nuclear localization and expression of HSP-4, HSP-6, HSP-60, GST-4 and SOD-3 in mutant nematode. Table S6. Effect of TRG on resistance to bacteria, high temperature and oxidation in wild-type nematode (N2). Table S7. Effects of TRG on age-related diseases. Table S8. Effect of TRG on gene expression at mRNA level in nematode. Table S9. Primers used for the analysis of mRNA expression levels in nematode. (Supplementary Materials)

\section{References}

[1] C. López-Otín, M. A. Blasco, L. Partridge, M. Serrano, and G. Kroemer, "The hallmarks of aging," Cell, vol. 153, no. 6, pp. 1194-1217, 2013.

[2] S. A. Wani and P. Kumar, "Fenugreek: a review on its nutraceutical properties and utilization in various food products," Journal of the Saudi Society of Agricultural Sciences, vol. 17, no. 2, pp. 97-106, 2018. 
[3] N. Mohamadi, F. Sharififar, M. Pournamdari, and M. Ansari, "A review on biosynthesis, analytical techniques, and pharmacological activities of trigonelline as a plant alkaloid," Journal of Dietary Supplements, vol. 15, no. 2, pp. 207-222, 2018.

[4] K. Pravalika, D. Sarmah, H. Kaur et al., "Trigonelline therapy confers neuroprotection by reduced glutathione mediated myeloperoxidase expression in animal model of ischemic stroke," Life Sciences, vol. 216, pp. 49-58, 2019.

[5] Z. Qiu, K. Wang, C. Jiang et al., "Trigonelline protects hippocampal neurons from oxygen-glucose deprivation- induced injury through activating the PI3K/Akt pathway," ChemicoBiological Interactions, vol. 317, p. 108946, 2020.

[6] S. Ilavenil, D. H. Kim, Y. I. Jeong et al., "Trigonelline protects the cardiocyte from hydrogen peroxide induced apoptosis in H9c2 cells," Asian Pacific Journal of Tropical Medicine, vol. 8, no. 4, pp. 263-268, 2015.

[7] A. N. Lone, A. T. Malik, H. S. Naikoo, R. S. Raghu, and S. A. Tasduq, "Trigonelline, a naturally occurring alkaloidal agent protects ultraviolet-B (UV-B) irradiation induced apoptotic cell death in human skin fibroblasts via attenuation of oxidative stress, restoration of cellular calcium homeostasis and prevention of endoplasmic reticulum (ER) stress," Journal of Photochemistry and Photobiology. B, vol. 202, p. 111720, 2020.

[8] H. Omidi-Ardali, Z. Lorigooini, A. Soltani, S. Balali-Dehkordi, and H. Amini-Khoei, "Inflammatory responses bridge comorbid cardiac disorder in experimental model of IBD induced by DSS: protective effect of the trigonelline," Inflammopharmacology, vol. 27, no. 6, pp. 1265-1273, 2019.

[9] M. Pournamdari, A. Mandegary, F. Sharififar et al., "Antiinflammatory subfractions separated from acidified chloroform fraction of Fenugreek Seeds (Trigonella foenum-graecumL.)," Journal of Dietary Supplements, vol. 15, no. 1, pp. 98-107, 2018.

[10] M. Khalili, M. Alavi, E. Esmaeil-Jamaat, T. Baluchnejadmojarad, and M. Roghani, "Trigonelline mitigates lipopolysaccharide-induced learning and memory impairment in the rat due to its anti-oxidative and antiinflammatory effect," International Immunopharmacology, vol. 61, pp. 355-362, 2018.

[11] O. Yoshinari, H. Sato, and K. Igarashi, "Anti-diabetic effects of pumpkin and its components, trigonelline and nicotinic acid, on Goto-Kakizaki rats," Bioscience, Biotechnology, and Biochemistry, vol. 73, no. 5, pp. 1033-1041, 2009.

[12] A. E. van Dijk, M. R. Olthof, J. C. Meeuse, E. Seebus, R. J. Heine, and R. M. van Dam, "Acute effects of decaffeinated coffee and the major coffee components chlorogenic acid and trigonelline on glucose tolerance," Diabetes Care, vol. 32, no. 6, pp. 1023-1025, 2009.

[13] H. V. Kamble and S. L. Bodhankar, "Antihyperglycemic activity of trigonelline and sitagliptin in nicotinamide- streptozotocin induced diabetes in Wistar rats," Biomed Agiicine andng Pathology, vol. 3, no. 3, pp. 125-130, 2013.

[14] A. D. Nugrahini, M. Ishida, T. Nakagawa, K. Nishi, and T. Sugahara, "Trigonelline: an alkaloid with anti-degranulation properties," Molecular Immunology, vol. 118, pp. 201-209, 2020.

[15] J. C. Liao, K. T. Lee, B. J. You et al., "Raf/ERK/Nrf 2 signaling pathway and MMP-7 expression involvement in the trigonelline-mediated inhibition of hepatocarcinoma cell migration," Food \& Nutrition Research, vol. 59, no. 1, article e29884, 2015.
[16] A. Arlt, S. Sebens, S. Krebs et al., "Inhibition of the Nrf2 transcription factor by the alkaloid trigonelline renders pancreatic cancer cells more susceptible to apoptosis through decreased proteasomal gene expression and proteasome activity," Oncogene, vol. 32, no. 40, pp. 4825-4835, 2013.

[17] M. Lu, L. Tan, X. G. Zhou et al., "Secoisolariciresinol diglucoside delays the progression of aging-related diseases and extends the lifespan of Caenorhabditis elegans via DAF-16 and HSF-1," Oxidative Medicine and Cellular Longevity, vol. 2020, Article ID e1293935, 2020.

[18] C. Rety, R. Gilbin, and E. Gomez, "Induction of reactive oxygen species and algal growth inhibition by tritiated water with or without copper," Environmental Toxicology, vol. 27, no. 3, pp. 155-165, 2012.

[19] N. Sitte, M. Huber, T. Grune et al., "Proteasome inhibition by lipofuscin/ceroid during postmitotic aging of fibroblasts," FASEB Journal, vol. 14, no. 11, pp. 1490-1498, 2000.

[20] D. E. Shore, C. E. Carr, and G. Ruvkun, "Induction of cytoprotective pathways is central to the extension of lifespan conferred by multiple longevity pathways," PLoS Genetics, vol. 8, no. 7, article e1002792, 2012.

[21] B. N. Dugger and D. W. Dickson, "Pathology of neurodegenerative diseases," Cold Spring Harbor Perspectives in Biology, vol. 9, no. 7, p. a028035, 2017.

[22] G. Murtaza, A. K. Khan, R. Rashid, S. Muneer, S. M. F. Hasan, and J. Chen, "FOXO transcriptional factors and long-term living," Oxidative Medicine and Cellular Longevity, vol. 2017, 8 pages, 2017.

[23] J. Tullet, J. W. Green, C. Au et al., "The SKN-1/Nrf2 transcription factor can protect against oxidative stress and increase lifespan inC. elegansby distinct mechanisms," Aging Cell, vol. 16, no. 5, pp. 1191-1194, 2017.

[24] W. C. Chiang, T. T. Ching, H. C. Lee, C. Mousigian, and A. L. Hsu, "HSF-1 regulators DDL-1/2 link insulin-like signaling to heat-shock responses and modulation of longevity," Cell, vol. 148, no. 1-2, pp. 322-334, 2012.

[25] G. Barja, "Updating the mitochondrial free radical theory of aging: an integrated view, key aspects, and confounding concepts," Antioxidants \& Redox Signaling, vol. 19, no. 12, pp. 1420-1445, 2013.

[26] S. Yanase, K. Yasuda, and N. Ishii, “Adaptive responses to oxidative damage in three mutants of_Caenorhabditis elegans_( _age-1_,_mev-1_and_daf-16_) that affect life span," Mechanisms of Ageing \& Development, vol. 123, no. 12, pp. 15791587, 2002.

[27] H. J. Weir, P. Yao, F. K. Huynh et al., "Dietary restriction and AMPK increase lifespan via mitochondrial network and peroxisome remodeling," Cell Metabolism, vol. 26, no. 6, pp. 884-896.e5, 2017.

[28] J. Zhou, L. Chan, and S. Zhou, “Trigonelline: a plant alkaloid with therapeutic potential for diabetes and central nervous system disease," Current Medicinal Chemistry, vol. 56, pp. 333$345,2012$.

[29] C. T. Murphy and P. J. Hu, "Insulin/insulin-like growth factor signaling in C. elegans," Worm Book, vol. 26, pp. 1-43, 2013.

[30] S. C. Johnson, P. S. Rabinovitch, and M. Kaeberlein, "mTOR is a key modulator of ageing and age-related disease," Nature, vol. 493, no. 7432, pp. 338-345, 2013.

[31] A. Kurtishi, B. Rosen, K. S. Patil, G. W. Alves, and S. G. Møller, "Cellular proteostasis in neurodegeneration," Molecular Neurobiology, vol. 56, no. 5, pp. 3676-3689, 2019. 